\title{
Elaboración de pan multigrano con harinas precocidas por extrusión
}

\section{Multigrain bread processing with extruded flours}

\author{
Crosa, María José (1), Tihista, Hugo ${ }^{(3)}$, Próspero, María Inés ${ }^{(3)}$, Cadenazzi, Mónica ${ }^{(4)}$, Souto, Laura (1), Curucthet, Ana ${ }^{(1)}$, \\ Ferreyra, Verónica (2), Suburú, Gabriela ${ }^{(1)}$, Escudero, Jorge ${ }^{(4)}$. \\ (1) Laboratorio Tecnológico del Uruguay, LATU - (2) Instituto Nacional de Tecnología Industrial, Centro Cereales y Oleaginosas, \\ 9 de Julio, Buenos Aires, Argentina - (3) Centro de Panaderos del Uruguay - (4) Cooperativa Agraria Nacional Uruguay - (4) \\ Consultor independiente, Laboratorio Tecnológico del Uruguay, LATU.
}

Contacto:mjcrosa.b@gmail.com

RECIBIDO: 16/5/2014 - APROBADO: 31/10/2014

\begin{abstract}
Resumen
Se estudió el efecto en la calidad del pan según el proceso de elaboración y del nivel de sustitución (20\%,36\%) de una harina precocida por extrusión preparada en base a combinación de avena, soja y salvado de trigo en la calidad del pan. Se caracterizó la harina compuesta según sus propiedades funcionales (Î́ndice de Absorción de Agua), granulometría y propiedades nutricionales (proteína, fibra total, fibra soluble, cenizas, materia grasa). Se realizó seguimiento del volumen, índice de blancura, medidas reológicas (dureza, cohesividad, elasticidad y masticabilidad) de los panes. Las condiciones del proceso de elaboración fue lo que generó mayor impacto en la calidad del pan. Los cambios operativos realizados provocaron un incremento del $37 \%$ en el volumen, $6 \%$ de elasticidad y $15 \%$ de cohesividad, y una disminución del $44 \%$ de dureza y $34 \%$ de masticabilidad. Esta tendencia se mantuvo en los cuatro días siguientes al día de elaboración. El nivel de sustitución de harina compuesta no provocó cambios significativos en la dureza, elasticidad y masticabilidad del pan, pero se observaron cambios en la cohesividad y volumen. El pan con $36 \%$ de sustitución resultó un $7 \%$ menos cohesivo y con un $27 \%$ menos de volumen que el de $20 \%$ de sustitución.

Palabras clave: Calidad pan multigrano, panificación, harinas compuestas.
\end{abstract}

\begin{abstract}
The effect in bread quality of a new bread making process and two replacement levels (20\%, 36\%) of refined wheat flour by extrusion precooked prepared based on combination of oats, soybeans and wheat bran was studied. Composite flour was characterized according to its functional properties (water absorption index), grain size and nutritional properties (protein, total fiber, soluble fiber, ash, fat). The volume, whiteness index, rheological measurements (hardness, cohesiveness, springiness and chewiness) of the bread were monitored. No significant changes were recorded in hardness, elasticity and chewiness of bread according to the level of substitution of composite flour; bread with $36 \%$ substitution was $7 \%$ less cohesive, with $27 \%$ less volume bread with $20 \%$ substitution. The process conditions caused greatest impact on the quality of bread. The new process resulted in a $37 \%$ increase in volume, $6 \%$ elasticity, $15 \%$ of cohesiveness and $44 \%$ decrease in hardness and $34 \%$ in chewiness, compared to the traditional process. This trend continued the four days following the date of processing. The substitution level of composite flour did not cause significant changes in hardness, elasticity and chewiness of bread, but changes were observed in cohesiveness and volume. Bread with $36 \%$ substitution was $7 \%$ less cohesive, with $27 \%$ less volume than the $20 \%$ substitution.

Keywords: Quality multigrain bread, bread process, composite flours.
\end{abstract}

\section{Introducción}

Es conocido a nivel mundial el beneficio del consumo de fibra en la dieta. En los últimos años, esta premisa fue difundida con mayor intensidad y diversos estudios fueron desarrollados para aumentar su integración en la dieta. El comité asesor de las Guías Alimentarias de Estados Unidos (U.S. Department of Agriculture, 2010) ha concluido que existe evidencia científica de que el consumo del grano entero reduce el riesgo de enfermedades cardiovasculares, obesidad y ganancia de peso. El Instituto de Medicina de Washington DC (OIM, 2011) también concluyó que el incremento del consumo de fibra dietética en niños provoca la extensión de vida del adulto mayor.

En Uruguay, según los datos aportados por la última Encuesta Nacional de Gastos e Ingresos del Instituto Nacional de Estadística (2005), la ingesta de fibra en los hogares urbanos se encuentra entre 8 gramos por día en la población con mayores ingresos y 3 gramos por día en la población con menores ingresos. Este bajo consumo es 
una situación que se extiende a nivel mundial. A pesar de los esfuerzos del Departamento de Agricultura de Estados Unidos (USDA) para estimular el consumo de 48 gramos por día de grano entero en los ciudadanos de Estados Unidos, las Guías Alimentarias (U.S. Department of Agriculture, 2010) informaron que el consumo medio del adulto es de 8 gramos por día. Menos del 1\% de los ciudadanos de Estados Unidos consumen la cantidad recomendada, el $20 \%$ no consume grano entero (Marquart, et al., 2013). Los británicos consumen 14 a 16 gramos por día, mientras que la población adulta de Suecia consume 42 gramos por día de grano entero y los daneses consumen 36 gramos por día (Thane, et al., 2007; Kyro, et al., 2012).

Entre las barreras para aumentar el consumo del grano entero se encuentran las preferencias sensoriales de los consumidores, la falta de información en la identificación de los alimentos de grano entero, la accesibilidad, el precio de las harinas de grano entero y el tiempo de preparación de los alimentos de grano entero (Kuznesof, et al., 2012; Ellis, et al., 2005; Lafferty, et al., 2006; Burgess-Champoux, et al., 2006).

El desarrollo de tecnologías para el procesamiento del grano entero que logre un alimento "palatable» y preserve sus propiedades nutricionales es un aspecto fundamental también para favorecer su consumo (Marquart, et al., 2013). Su desarrollo comercial ha sido acotado por la vida útil más corta comparada con las harinas refinadas. El grano entero posee un contenido natural de grasas, aceites y de enzimas lipolíticas que dificultan su incorporación en los alimentos. Crosa et al. (2013), Hansen y Rose (1996), Pomeranz et al. (1992) y Tait et al. (1988) estudiaron la disminución de la calidad del pan debido al enranciamiento de las harinas.

La extrusión fue clasificada como una tecnología de cocción HTST (alta temperatura, corto tiempo) que provoca cambios químicos y físicos deseables en el alimento: destruye o inactiva componentes antinutricionales o tóxicos (inhibidores de tripsina, hematoglutininas, etcétera), enzimas indeseables como la lipoxigenasa, peroxidasa y los microorganismos. A nivel nutricional, el proceso minimiza la degradación de los nutrientes por calor y mejora la digestibilidad por gelatinización del almidón y desnaturalización de la proteína, comparado con los procesos térmicos tradicionales (Crosa, et al., 2013; Robin, et al. 2012; Sosa-Moguel, et al., 2009; Athar, et al., 2006; Brennan, et al., 2011; Killeit, 1994; Turgeon, et al., 2011; Rose, 2008; Sing, et al., 2007; Vetrimani, 1990). El proceso de cocción durante la extrusión provoca cambios en la estructura granular del almidón que afectan las propiedades de hidratación. Según el grado de cocción, ocurre un incremento de la proporción de sólidos dispersables y de restos de gránulos hinchados que determinarán las características reológicas de la dispersión de la harina precocida (Pérez, et al., 2008). La mayor absorción de agua de las harinas extrudidas en relación a las no extrudidas es un aspecto a considerar para su uso en la elaboración del pan.

El pan es un producto "pronto para el consumo» de consumo masivo en la población uruguaya. De acuerdo a la Encuesta Nacional de Gastos e Ingresos del Instituto Nacional de Estadística (2005), el consumo es de 150 gramos promedio por día de pan elaborado con harina de trigo refinada, siendo despreciable el consumo de pan integral o en base a otros cereales. La sustitución parcial de harina refinada por harina multigrano aporta al pan una mejora nutricional por el aumento de aminoácidos esenciales, fi- bra, minerales y vitaminas. La soja posee proteínas de alta calidad (41\%) particularmente rica en lisina, $27 \%$ de fibra total correspondiente a la fracción insoluble, la avena con un $16 \%$ de proteínas, $21,3 \%$ de fibra total, del cual 3,3\% corresponde a fibra soluble. Esta fracción soluble se destaca por su contenido de betaglucanos con importantes beneficios nutricionales: aumentar el contenido gastrointestinal, interferir en la formación de micelas y absorción de lípidos, aumentar la excreción de esteroles y ácidos biliares e inhibir la síntesis de colesterol hepático debido a la absorción del ácido propiónico formado en la fermentación (Matos-Chamorro, 2010). El salvado presenta un alto contenido de fibra total $(43,4 \%)$, correspondiente a la fracción insoluble.

La inclusión de harinas multigrano en la masa del pan puede provocar una pérdida de su calidad sensorial si no se estudian adecuadamente las condiciones operativas del proceso de panificación. Durante el amasado e hidratación de las proteínas del trigo gluteninas y gliadinas se da lugar a la formación de una red tridimensional, lograda por la polimerización de los enlaces disulfuro entre restos del aminoácido cisteína y otros enlaces que colaboran en la formación de la estructura. En esta etapa el agua se encuentra fuertemente retenida por esta estructura y pasa a formar parte de la misma. La formación de esta red requiere que el trabajo mecánico de amasado prosiga hasta lograr el máximo de enlaces posibles. Esta condición es sustancial para conseguir la mayor retención de los gases formados durante la fermentación dentro de la masa y la que permitirá su esponjamiento.

Cuando en la composición de la masa hay presencia de elementos integrales como granos enteros o parcialmente molidos desde el inicio del amasado, la formación de la red se dificulta, quedando interrumpida en partes, y la masa se debilita provocando defectos en el producto final, como la disminución del volumen y el aumento de la dureza de la miga del pan multigrano (Indrani, et al., 2010 y 2011; Gómez, et al., 2011; Feillet, et al.,1996).

En este trabajo se proponen cambios en la cantidad y el momento de agregado del agua respecto a la receta habitual, tomando en cuenta los cambios en las propiedades funcionales de la harina compuesta precocida por extrusión. El objetivo de este artículo es estudiar el efecto en la calidad del pan elaborado según el nuevo proceso.

\section{Materiales y Métodos}

A continuación se describen los análisis fisicoquímicos utilizados para caracterizar la harina compuesta, las medidas de calidad del pan resultante, la caracterización de los procesos de elaboración de harinas y de los panes. Se detallan también los procedimientos experimentales utilizados.

\section{Análisis fisicoquímicos de las harinas}

Fueron realizados siete tipos de análisis fisicoquímicos en la harina compuesta.

- Granulometría, tamizado en equipo Retsch, con Tamices ASTM \# 18, \#60 y base, en amplitud 60 durante 10 minutos. Medida por triplicado.

- Proteínas, expresada en gramos de proteína en $100 \mathrm{~g}$ de harina según AACC 46-30, método de combustión en equipo Leco FP-528. Medida por duplicado. 
- Fibra total e insoluble, expresada en gramos de fibra en 100 g de harina según: Método AOAC 985.29 (AOAC, 2010). Medida por duplicado.

- Cenizas, según ISO 2171 (International Organization for Standardization, 2010). Medida por duplicado.

- Humedad (\%H), expresada en gramos de agua en $100 \mathrm{~g}$ de harina. En la harina de soja se siguió el protocolo de análisis basado en AOCS Bc 2-49. En el resto de las harinas la determinación de humedad fue en base a la técnica AACC 44-40. Medida por duplicado.

- Materia grasa (\%MG), expresada en gramos de materia grasa en $100 \mathrm{~g}$ de materia seca de la harina. En equipo Soxtec de Tecator, solvente éter de petróleo ppa., se expresa en g/100 g de muestra de harina, luego de determinar el tiempo óptimo de extracción de materia grasa para cada harina. Basado en AOCS Bc 3-49. Medida por duplicado.

- Medida del índice de absorción de agua (IAA) según Anderson et al. (1969). Se pesa 2,5 g de muestra en tubos de centrífuga de $50 \mathrm{~mL}$ y se le agregan $30 \mathrm{~mL}$ de agua destilada, se termostatiza en baño de agua a $30^{\circ} \mathrm{C}$ durante 30 minutos, agitándolos cada 5 minutos en un vortex durante 15 segundos. Luego se centrifuga a temperatura ambiente, a $5000 \mathrm{rpm}$ por 30 minutos. Se separa el sobrenadante y se pesa el gel. El IAA se registra como el peso del gel (g) / peso de muestra (g). La medida se realiza por triplicado.

\section{Medidas de calidad del pan}

Se analizaron cuatro variables para medir la calidad del pan: color, volumen, textura de la miga por ensayo de TPA y escaneado de la imagen del pan resultante. La medida se realiza por triplicado.

- Medida del color. Se realizó en colorímetro HUNTERLAB PLUS XE. Las medidas de color fueron expresadas por los valores especiales obtenidos por el sistema CIE$\mathrm{L}^{*} \mathrm{a}^{*} \mathrm{~b}^{*}$; el parámetro $\mathrm{L}^{*}$ describe la luminosidad en una escala de 0 (negro) a 100 (blanco puro), el parámetro $\mathrm{a}^{*}$ es matiz del color (+ rojo - verde), y el parámetro b* es matiz del color (+ amarillo - azul). Se determinó el índice de blancura (WI), acorde a Hsu et al. (2003). La medida se realiza por triplicado.

$$
\mathrm{WI}=100-\operatorname{RAIZ}\left((100-\mathrm{L})^{\star}(100-\mathrm{L})+\mathrm{a}^{\star} \mathrm{a}+\mathrm{b}^{\star} \mathrm{b}\right)
$$

- Medida del volumen. Se realizó por método de desplazamiento de semillas de canola. Se llena totalmente un recipiente plástico de 2,0 L, con semillas de canola, se enrasa y se registra el peso de las semillas. Se coloca el pan en el recipiente, se completa con semillas y se vuelve a enrasar. Se mide el volumen de las semillas que quedaron en la probeta y se registra ese volumen como el volumen del pan. La medida se realiza por triplicado.

- Ensayo de TPA (Texture Analysis Profile). Ensayo con texturómetro TA.XT2 usando un cilindro de $36 \mathrm{~mm}$ con bordes redondeados, en las siguientes condiciones de medida: Test speed: $1 \mathrm{~mm} / \mathrm{sec}, 40 \%$ deformación en $2,43 \pm 0,02 \mathrm{~cm}$ (dos rodajas) y 5 segundos de relajación. Se realizaron seis repeticiones en cada ensayo. Las muestras se cortaron en rebanadas de $2,5 \mathrm{~cm}$ de

\begin{tabular}{|l|c|c|c|c|}
\hline Nivel de sustitución & \multicolumn{2}{|c|}{ N1: 20\% } & \multicolumn{2}{c|}{ N2: 36\% } \\
\hline Ingredientes & $\%$ & Gramos & $\%$ & Gramos \\
\hline $\begin{array}{l}\text { Harina de trigo panadera } \\
\text { (000) }\end{array}$ & 80 & 1200 & 64 & 960 \\
Harina compuesta & 20 & 300 & 36 & 540 \\
Aceite & 3 & 45 & 3 & 45 \\
Agua masa blanca & 48 & 720 & 38,4 & 576 \\
$\begin{array}{l}\text { Agua prehidratación } \\
\text { Sal }\end{array}$ & 20 & 300 & 36 & 540 \\
$\begin{array}{l}\text { Levadura seca instantánea } \\
\text { (Lesaffre Group) }\end{array}$ & 1,4 & 21 & 1,4 & 21 \\
$\begin{array}{l}\text { Gluten vital } \\
\begin{array}{l}\text { Sp+ (mejorador } \\
\text { de pan Nutrigold: } \\
\text { Super pan plus) }\end{array}\end{array}$ & 4 & 60 & 4 & 60 \\
\hline
\end{tabular}

Tabla 1. Formulación del pan en los dos niveles de sustitución de harina precocida por extrusión.

altura, la medida se realizó en el centro de la rebanada. El valor de la fuerza máxima del ensayo se interpretó como la dureza de la miga de pan; la recuperación de la altura de las rodajas luego de ser comprimidas una vez se asoció con la elasticidad; la relación entre las dos áreas (a2/a1) con la cohesividad, y la masticabilidad del pan se interpretó como el resultado del producto de la dureza, la cohesividad y la elasticidad (Bourne, 2002).

- Imagen escaneada. Se escanea una rodaja de $1,5 \mathrm{~cm}$ de espesor de pan. La medida se realiza por triplicado.

\section{Proceso de elaboración de las harinas}

Las harinas fueron elaboradas en la planta semi-industrial del Centro Cereales y Oleaginosas del Instituto Nacional de Tecnología Industrial (9 de Julio, Buenos Aires, Argentina). Los granos de avena, sorgo y soja fueron clasificados, limpios y molidos en molino de martillo hasta un tamaño de partícula inferior a $4 \mathrm{~mm}$. El germen y salvado no se molieron previo a la extrusión. Fueron procesados en una extrusora de tornillo simple de tres secciones con las siguientes especificaciones: longitud del barril $200 \mathrm{~mm}$; diámetro del barril $105 \mathrm{~mm}$; velocidad de tornillo de $60 \mathrm{rpm}$. La velocidad de alimentación de la extrusora fue en promedio $550 \mathrm{~kg} / \mathrm{h}$ y el tiempo de residencia promedio del producto en la misma fue de tres segundos. En el procesamiento de la soja y el germen, la torta obtenida luego de la extrusión se desgrasó parcialmente mediante prensa-tornillo. En la siguiente etapa se procedió al enfriado y secado de las harinas mediante una corriente de aire que es forzada a pasar a través de la columna de secado. Por último, se llevó a cabo la molienda final en molino de martillo hasta la granulometría deseada para cada harina. Todos los productos finales fueron envasados en bolsas de papel Kraft (doble lámina) con lámina interna de polietileno. 


\section{Proceso de elaboración de pan}

Los ingredientes del pan fueron: $1500 \mathrm{~g}$ en total de harina (mezcla de harina refinada-harina precocida por extrusión), aceite (45 g), sal (1,5 g), levadura seca instantánea (1,4 g), gluten vital (4 g), mejorador de pan $(0,8 \mathrm{~g})$.

Se elaboró pan con dos niveles de sustitución de harina precocida por extrusión por harina refinada, N1: $20 \%$ y N2: $36 \%$. El volumen de agua agregado en cada receta varió según la relación harina refinada y compuesta. Se mantuvo la misma relación de peso de agua y peso de harina refinada (0.6:1) y la misma relación del agua con harina compuesta (1:1). En la Tabla 1 se describe la receta del pan según el nivel de sustitución de la harina refinada por la harina precocida por extrusión o harina compuesta.

\section{Ensayo de panificación}

Las diferencias entre los procesos de elaboración de pan consistieron en la forma en que se combina el agua con los ingredientes y en el momento en que se agregó la harina compuesta durante la panificación. A continuación se describen:

- Descripción procedimiento P1: Se mezclaron los ingredientes secos, incluyendo la harina compuesta, menos la levadura. Se agregó el $80 \%$ del agua total y se amasó en velocidad lenta ( $123 \mathrm{rev} / \mathrm{min}$ de batidor y $12 \mathrm{rev} / \mathrm{min}$ del tacho), una vez hidratados los ingredientes se agregó el aceite y el resto del agua amasando en velocidad lenta hasta completar los 5 minutos. Se continuó amasando en velocidad rápida $(185 \mathrm{rev} / \mathrm{min}$ de batidor y $17 \mathrm{rev} / \mathrm{min}$ del tacho) y pasados $2 \mathrm{minu}$ tos se agregó la levadura. Una vez que la masa quedó elástica se interrumpió el amasado y se dejó descansar por 30 minutos (F1) el amasijo. Pasado este tiempo, se pesaron bollos de $198 \mathrm{~g}$, se dejaron descansar por 15 minutos (FI), se armaron y se estibaron. Se realizó la fermentación final en cámara $\left(35^{\circ} \mathrm{C}\right.$ y $80 \%$ HR) durante 75 minutos. Luego se cocinaron en horno a $200^{\circ} \mathrm{C}$ por 25 minutos, se desmoldaron una vez sacado del horno y se dejaron enfriar por 2 horas.

- Descripción procedimiento P2: La elaboración de pan mediante este procedimiento presentó dos etapas. En una primera instancia se elaboró una masa blanca, homogénea y elástica y posteriormente se agregaron las harinas compuestas, prehidratadas como se detalla a continuación. En ambos procesos se incorpora la misma cantidad de agua en total que en el procedimiento P1. Se prehidrató la harina compuesta en proporción Harina: Agua de 1:1 con el inicio del proceso de elaboración. Se mezclaron los ingredientes secos menos la levadura y la harina compuesta, se agregaron el $80 \%$ del agua para la masa blanca y se amasaron a velocidad lenta. Una vez hidratados, se agregó el aceite, el resto del agua y se continuó amasando hasta completar los 5 minutos. Luego se amasó a velocidad rápida y pasados 2 minutos se incorporó la levadura. Una vez formado el gluten y al haber obtenido una masa elástica se interrumpió el amasado y se dejó descansar el amasijo por 30 minutos. Cumplido el descanso de la masa blanca, se unieron las harinas prehidratadas amasando un minuto en velocidad lenta y dos minutos en velocidad rápida. Pasado este tiempo se dejó descansar el amasijo en bloque por 15 minutos, para luego pesar bollos de 198 g y se dejó descansar por 15 minutos más. Se armaron, se estibaron y luego se realizó la fermentación final en cámara $\left(35^{\circ} \mathrm{C}\right.$ y $80 \% \mathrm{HR}$ ) durante 75 minutos. Por último se cocinó en horno a $200{ }^{\circ} \mathrm{C}$ durante 25 minutos, se desmoldó y se dejó enfriar por dos horas.

\section{Diseño estadístico}

- Comparación del procedimiento de elaboración y nivel de sustitución. Se realizó un diseño completamente aleatorizado (DCA). Los tratamientos consistieron en un factorial de 2 niveles en proceso de elaboración (P1 y P2) y 2 niveles en sustitución de harina precocida por extrusión ( $20 \%$ y $36 \%)$. Cada tratamiento se realizó por duplicado y consistió en un amasijo (unidad experimental) del cual se obtuvieron 8 panes de 198 g. Se realizaron análisis de varianza para el modelo completo y las diferencias de medias fueron testeadas por el test «t» o diferencia mínima significativa (LSD). Las variables del ensayo fueron volumen, color, textura (ensayo TPA).

- Seguimiento de textura durante el almacenamiento. Se realizó el ensayo de textura TPA en unidades de pan provenientes de cada tratamiento durante 4 días consecutivos. Para cada tratamiento se calculó la media e intervalo de confianza en cada día. Los datos fueron analizados según un diseño completamente aleatorizado de 4 tratamientos, tomándose los días como repeticiones, considerando las medidas repetidas en el tiempo $\mathrm{y}$ ajustando una matriz de correlación temporal AR(1) (autoregresiva de orden 1) y CS (simétrica compuesta).

\section{Resultados}

\section{Caracterización fisicoquímica de la harina compuesta}

La harina compuesta fue formulada en base a avena, salvado y soja, dando lugar a una composición porcentual: $4,4 \%$ de cenizas, $9 \%$ humedad, $1,1 \%$ fibra soluble, $29,5 \%$ fibra insoluble, $5 \%$ materia grasa, $24,5 \%$ proteína.

El tamaño de grano de la harina se compone de las siguientes partes: $17 \%$ fue retenido por malla $1 \mathrm{~mm}$ (tamiz 18), $35,8 \%$ fue retenido por malla $0,42 \mathrm{~mm}$ (tamiz 40 ), $14 \%$ fue retenido por malla $0,25 \mathrm{~mm}$ (tamiz 60$), 15,8 \%$ fue retenido por malla $0,177 \mathrm{~mm}$ (tamiz 80 ) y el $17,5 \%$ quedó en la base.

La harina compuesta extrudida presentó un índice de absorción de agua de $5,8 \pm 0,2 \mathrm{~g}$ agua/g harina y $4,07 \pm 0,2 \mathrm{~g}$ en la harina sin extrusión de la misma granulometría a la extrudida.

\section{Comparación de los procesos en el pan recién elaborado}

Se presentan en la Figura 1 las imágenes obtenidas por escaneo y en la Tabla 2 los resultados de las medidas de textura, volumen y color del pan según los 4 tratamientos con un día de almacenamiento. 


\begin{tabular}{|c|c|c|c|c|c|c|c|c|c|c|c|c|c|c|c|c|c|}
\hline \multirow{3}{*}{ Factor } & & \multicolumn{8}{|c|}{ Textura } & \multirow{2}{*}{\multicolumn{2}{|c|}{ Volumen }} & \multicolumn{6}{|c|}{ Color } \\
\hline & & \multicolumn{2}{|c|}{ Dureza } & \multicolumn{2}{|c|}{ Elasticidad } & \multicolumn{2}{|c|}{ Cohesividad } & \multicolumn{2}{|c|}{ Masticabilidad } & & & \multicolumn{2}{|r|}{$\mathrm{L}$} & \multicolumn{2}{|c|}{ A } & \multicolumn{2}{|c|}{ B } \\
\hline & & Medias & $\begin{array}{c}\text { P-valor } \\
\text { DMS }\end{array}$ & Medias & $\begin{array}{c}\text { P-valor } \\
\text { DMS }\end{array}$ & Medias & $\begin{array}{c}\text { P-valor } \\
\text { DMS }\end{array}$ & Medias & $\begin{array}{l}\text { P-valor } \\
\text { DMS }\end{array}$ & Medias & $\begin{array}{c}\text { P-valor } \\
\text { DMS }\end{array}$ & Medias & $\begin{array}{c}\text { P-valor } \\
\text { DMS }\end{array}$ & Medias & $\begin{array}{c}\text { P-valor } \\
\text { DMS }\end{array}$ & Medias & $\begin{array}{c}\text { P-valor } \\
\text { DMS }\end{array}$ \\
\hline Proceso & $\begin{array}{l}\text { P1 } \\
\text { P2 }\end{array}$ & $\begin{array}{l}1117 \mathrm{a} \\
622 \mathrm{~b}\end{array}$ & $\begin{array}{c}0,0133 \\
324\end{array}$ & $\begin{array}{l}0,90 \mathrm{a} \\
0,94 \mathrm{~b}\end{array}$ & $\begin{array}{c}\mathbf{0 , 0 1 3 3} \\
0,022\end{array}$ & $\begin{array}{l}0,57 \mathrm{a} \\
0,66 \mathrm{~b}\end{array}$ & $\begin{array}{l}\mathbf{0 , 0 0 2 5} \\
0,0339\end{array}$ & $\begin{array}{l}6629 \mathrm{a} \\
4362 \mathrm{~b}\end{array}$ & $\begin{array}{c}\mathbf{0 , 0 2 0 2} \\
1686\end{array}$ & $\begin{array}{c}418 \mathrm{a} \\
573 \mathrm{~b}\end{array}$ & $\begin{array}{c}\mathbf{0 , 0 0 8 0} \\
88\end{array}$ & $\begin{array}{l}58,57 \mathrm{a} \\
59,85 \mathrm{~b}\end{array}$ & $\begin{array}{c}\mathbf{0 , 0 0 0 3} \\
0,30\end{array}$ & $\begin{array}{l}5,22 \mathrm{a} \\
5,09 \mathrm{a}\end{array}$ & $\begin{array}{c}\mathbf{0 , 0 5 8 2} \\
0,14\end{array}$ & $\begin{array}{l}15,25 \mathrm{a} \\
14,96 \mathrm{a}\end{array}$ & $\begin{array}{c}\mathbf{0 . 1 2 3 1} \\
0,41\end{array}$ \\
\hline \%sustitución & $\begin{array}{l}20 \\
36\end{array}$ & $\begin{array}{l}760 \mathrm{a} \\
979 \mathrm{a}\end{array}$ & $\begin{array}{c}\mathbf{0 , 1 3 4 9} \\
324\end{array}$ & $\begin{array}{l}0,92 \mathrm{a} \\
0,92 \mathrm{a}\end{array}$ & $\begin{array}{c}\mathbf{0 , 3 3 9 0} \\
0,022\end{array}$ & $\begin{array}{l}0,64 \mathrm{a} \\
0,59 \mathrm{~b}\end{array}$ & \begin{tabular}{l|}
$\mathbf{0 , 0 2 1 3}$ \\
0,0339
\end{tabular} & $\begin{array}{l}5004 \mathrm{a} \\
5987 \mathrm{a}\end{array}$ & $\begin{array}{c}\mathbf{0 , 1 8 0 8} \\
1686\end{array}$ & $\begin{array}{l}575 \mathrm{a} \\
416 \mathrm{~b}\end{array}$ & $\begin{array}{c}\mathbf{0 , 0 0 7 4} \\
88\end{array}$ & $\begin{array}{l}62,23 \mathrm{a} \\
56,19 \mathrm{~b}\end{array}$ & $\begin{array}{c}<\mathbf{0 , 0 0 0 1} \\
0,30\end{array}$ & $\begin{array}{l}4,62 \mathrm{a} \\
5,69 \mathrm{~b}\end{array}$ & $\mid \begin{array}{c}<\mathbf{0 , 0 0 0 1} \\
0,14\end{array}$ & $\begin{array}{l}15,02 \mathrm{a} \\
15,19 \mathrm{a}\end{array}$ & $\begin{array}{c}\mathbf{0 . 3 1 1 6} \\
0,41\end{array}$ \\
\hline \multicolumn{2}{|l|}{$\begin{array}{l}\text { Interacción: } \\
\text { Proceso } \\
\text { \%sustitución }\end{array}$} & \multicolumn{2}{|c|}{0,9447} & \multicolumn{2}{|c|}{0,2444} & \multicolumn{2}{|c|}{0,9473} & \multicolumn{2}{|c|}{0,9329} & \multicolumn{2}{|c|}{0,0874} & \multicolumn{2}{|c|}{$\begin{array}{l}\mathbf{0 , 0 3 0 9} \\
0,4288\end{array}$} & \multicolumn{2}{|c|}{$\begin{array}{l}\mathbf{0 , 0 3 4 2} \\
0,1978\end{array}$} & \multicolumn{2}{|c|}{0,1546} \\
\hline
\end{tabular}

Tabla 2. Medidas de volumen y color según los 4 tratamientos en pan con un día de elaboración. Los parámetros de textura informados en la tabla corresponden a la interpretación de la curva durante la compresión del pan hasta un $40 \%$. Dureza corresponde a la fuerza máxima, elasticidad a la recuperación de la altura de las rodajas luego de ser comprimidas una vez, cohesividad a la relación entre las dos áreas (a2/a1) y masticabilidad se calculó como el producto de la dureza, la cohesividad y la elasticidad. Se presentan las medias, Pvalor y DMS de cada variable analizada. Letras iguales entre valores indican que no existen diferencias significativas entre efectos con un $95 \%$ de confianza.

\begin{tabular}{|c|c|c|c|c|c|c|c|c|}
\hline \multicolumn{2}{|c|}{${\text { Interacción proceso }{ }^{*} \text { nivel de sustitución }}^{2}$} & \multicolumn{3}{c|}{ Variable estudio: a } & \multicolumn{3}{c|}{ Variable estudio: WI } \\
Proceso & \%sustitución & Medias & Proceso & \%sustitución & Medias & Proceso & $\%$ sustitución & Medias \\
P1 & 36 & $55,73 \mathrm{a}$ & $\mathrm{P} 2$ & 20 & $4,47 \mathrm{a}$ & $\mathrm{P} 1$ & 36 & $52,85 \mathrm{a}$ \\
$\mathrm{P} 2$ & 36 & $56,65 \mathrm{~b}$ & $\mathrm{P} 1$ & 20 & $4,77 \mathrm{~b}$ & $\mathrm{P} 2$ & 36 & $53,72 \mathrm{~b}$ \\
$\mathrm{P} 1$ & 20 & $61,41 \mathrm{c}$ & $\mathrm{P} 1$ & 36 & $5,67 \mathrm{c}$ & $\mathrm{P} 1$ & 20 & $58,21 \mathrm{c}$ \\
$\mathrm{P} 2$ & 20 & $63,05 \mathrm{~d}$ & $\mathrm{P} 2$ & 36 & $5,70 \mathrm{c}$ & $\mathrm{P} 2$ & 20 & $59,96 \mathrm{~d}$ \\
\hline
\end{tabular}

Tabla 3. Estudio del efecto de la interacción entre «proceso» con «nivel de sustitución» con 95\% de confianza.

P1

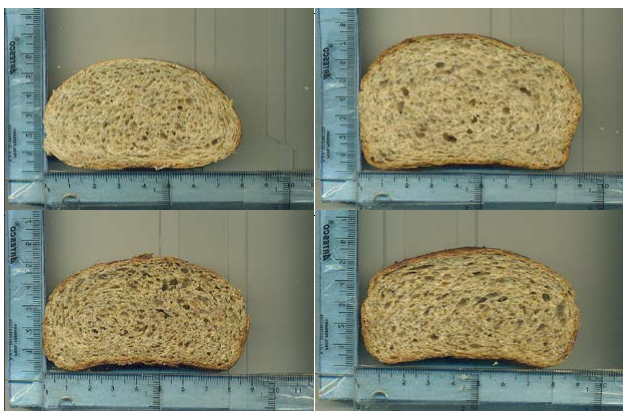

Figura 1. Imagen escaneada de una rodaja de pan en los 4 tratamientos. En las columnas se ubican los panes elaborados según el proceso P1 y P2. En las filas se ubican los panes elaborados con $20 \%$ y $36 \%$ de sustitución.

\section{Seguimiento de textura durante almacenamiento}

A continuación se presenta en forma gráfica la variación durante 4 días consecutivos de las medidas de compresión del pan. Cada medida se interpreta con un parámetro de textura: fuerza máxima (dureza), recuperación de la altura luego de la primera compresión (elasticidad), relación entre dos áreas (cohesividad) y masticabilidad como el producto de las tres mediciones. Se informan las medias y los intervalos de confianza (95\%) de estos parámetros por día en cada tratamiento (Gráficos 1, 2, 3 y 4).

En la Tabla 5 se presenta el ANOVA tomando el día de medida y considerando las repeticiones independientes para cada tratamiento. 


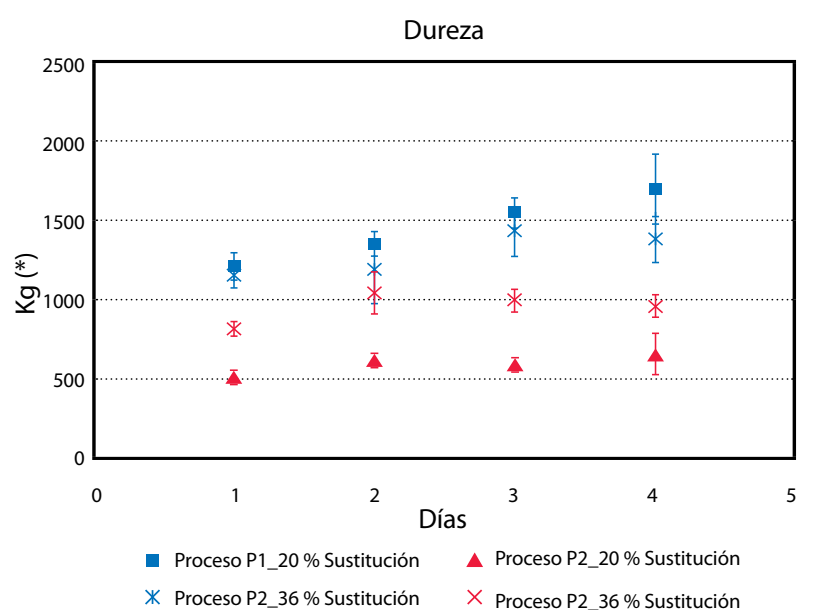

Gráfico 1. Medida de la fuerza máxima en el ensayo de compresión (TPA), interpretada como la dureza de la miga de pan, según el nivel sustitución de harina compuesta $(20 \%, 36 \%)$ y el proceso de elaboración (P1 y P2). Se presentan la media y el intervalo de confianza (95\%)

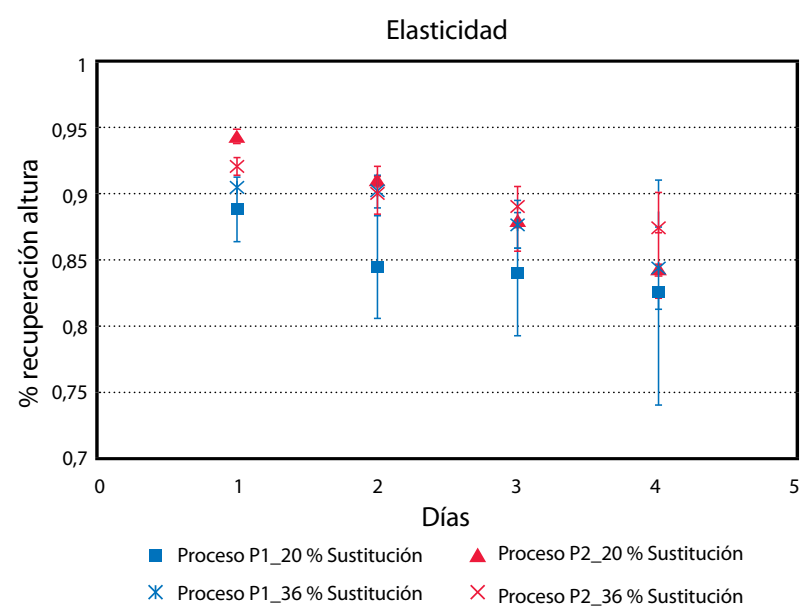

Gráfico 2. Medida del grado de recuperación de su altura luego de la primera compresión, asociada con la elasticidad de la miga del pan elaborado según el nivel sustitución de harina compuesta (20\%,36\%) y el proceso de elaboración (P1 y P2). Se presenta la media y el intervalo de confianza (95\%)

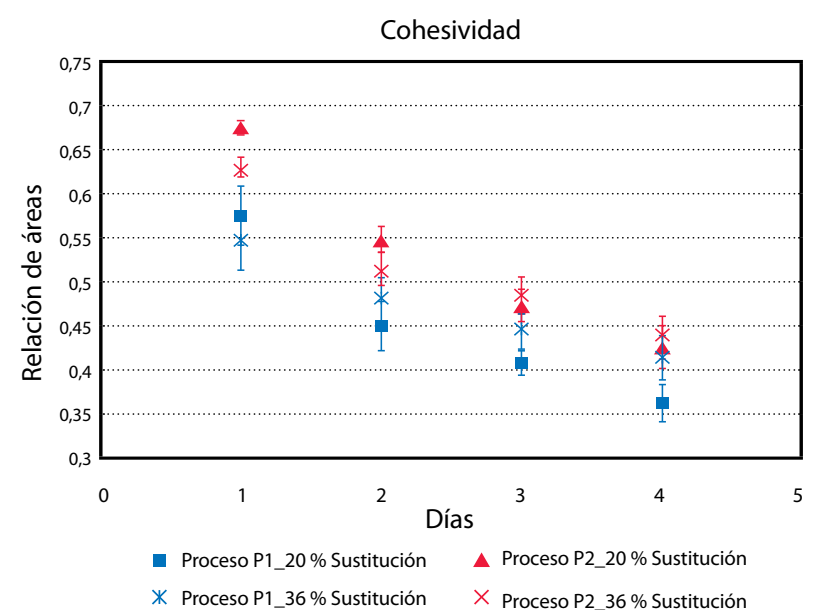

Gráfico 3. Relación de las áreas del ensayo de TPA, interpretado como un parámetro de cohesividad de la miga de pan elaborado con dos niveles de sustitución de harina compuesta $(20 \%, 36 \%)$ y dos procedimientos (P1 y P2). Se presenta la media y el intervalo de confianza (95\%)

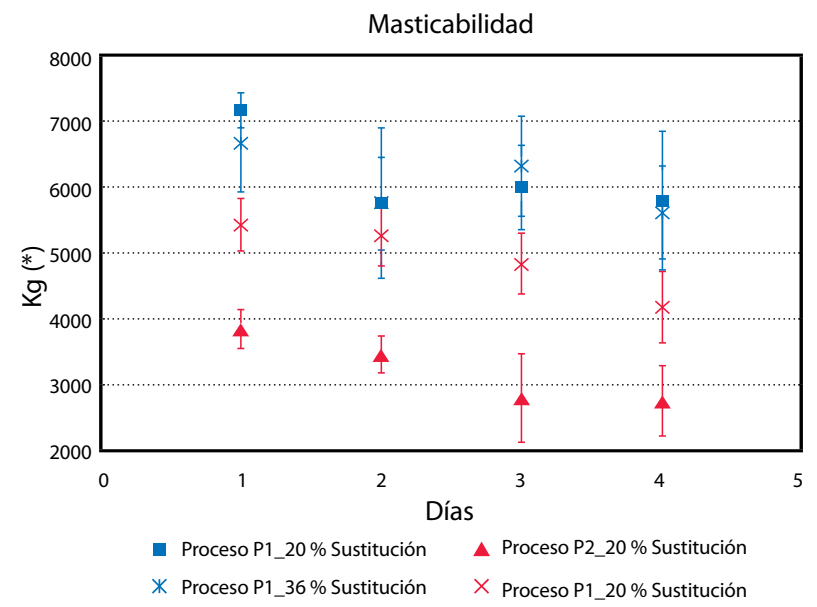

Gráfico 4. Cálculo de masticabilidad de la miga de pan, con dos niveles de sustitución de harina compuesta (20\%, 36\%) y dos procedimientos ( $\mathrm{P} 1$ y $\mathrm{P} 2$ ). Se presenta la media y el intervalo de confianza (95\%)

\begin{tabular}{|c|c|c|c|c|c|c|c|c|c|}
\hline \multirow{3}{*}{\multicolumn{2}{|c|}{ Factor }} & \multicolumn{8}{|c|}{ Textura } \\
\hline & & \multicolumn{2}{|r|}{ Dureza } & \multicolumn{2}{|c|}{ Elasticidad } & \multicolumn{2}{|c|}{ Cohesividad } & \multicolumn{2}{|c|}{ Masticabilidad } \\
\hline & & medias & P-valor Error std & medias & P-valor Error std & medias & P-valor Error std & medias & P-valor Error std \\
\hline Proceso & $\begin{array}{l}\text { P1 } \\
\text { P2 }\end{array}$ & $\begin{array}{l}1367 \mathrm{a} \\
775 \mathrm{~b}\end{array}$ & $<0,0001$ & $\begin{array}{l}0,8653 \mathrm{a} \\
0,8950 \mathrm{a}\end{array}$ & 0,0738 & $\begin{array}{l}0,4599 a \\
0,5224 a\end{array}$ & 0,1734 & $\begin{array}{l}6128 \mathrm{a} \\
4066 \mathrm{~b}\end{array}$ & $<0,0001$ \\
\hline $\begin{array}{l}\text { Nivel de } \\
\text { sustitución }\end{array}$ & $\begin{array}{l}20 \\
36\end{array}$ & $\begin{array}{l}1022 \mathrm{a} \\
1120 \mathrm{a}\end{array}$ & 0,1851 & $\begin{array}{c}0,8718 \mathrm{a} \\
0,885 \mathrm{a}\end{array}$ & 0,927 & $\begin{array}{l}0,4891 \mathrm{a} \\
0,4931 \mathrm{a}\end{array}$ & 0,9277 & $\begin{array}{l}4690 \mathrm{~b} \\
5504 \mathrm{a}\end{array}$ & 0,0139 \\
\hline \multicolumn{2}{|c|}{$\begin{array}{l}\text { Proceso }^{\star} \\
\text { Nivel sustitución }\end{array}$} & \multicolumn{2}{|r|}{0,0031} & \multicolumn{2}{|r|}{0,3201} & \multicolumn{2}{|r|}{0,6718} & \multicolumn{2}{|r|}{0,0080} \\
\hline
\end{tabular}

Tabla 4. Resumen de análisis de varianza, promedios de los efectos principales y la interacción en el seguimiento de la textura del pan. Letras iguales entre valores en columna indican diferencias no significativas entre los procesos o los porcentajes de incorporación para un nivel de significancia del $5 \%$. 


\begin{tabular}{|c|c|c|c|c|c|}
\hline \multicolumn{3}{|c|}{ Efecto interacción proceso - Nivel de sustitución } \\
Variable estudio: Dureza & \multicolumn{3}{c|}{ Variable estudio: Masticabilidad } \\
Proceso & Nivel de Sustitución & Medias & Proceso & Nivel de Sustitución & Medias \\
P1 & 20 & $1446 \mathrm{a}$ & P1 & 20 & $6170 \mathrm{a}$ \\
P1 & 36 & $1288 \mathrm{a}$ & P1 & 36 & $6086 \mathrm{ab}$ \\
P2 & 36 & $952 \mathrm{~b}$ & P2 & 36 & $4921 \mathrm{~b}$ \\
\hline P2 & 20 & $598 \mathrm{c}$ & P2 & 20 & $3211 \mathrm{c}$ \\
\hline
\end{tabular}

Tabla 5. Estudio del efecto de la interacción entre «proceso» con «\% sustitución» significativas (5\%).

\section{Discusión}

En la Figura 1 se presentan las imágenes obtenidas por scaneo del pan y se observa a simple vista el mayor desarrollo de los panes mediante el procedimiento $\mathrm{P} 2$ en relación al P1.

Según se aprecia en la Tabla 2, en las variables de textura $\mathrm{y}$ volumen el efecto de la interacción entre "proceso" $\mathrm{y}$ «nivel de sustitución» resultó no significativa, por lo cual en este caso se suma el impacto de los efectos (modelo aditivo). El estudio del efecto "proceso" presentó diferencias significativas en las variables que describen la textura del pan y en el volumen. El proceso $\mathrm{P} 1$ da panes significativamente más duros, menos elásticos, menos cohesivos, de mayor masticabilidad y menor volumen. Según el efecto «nivel de sustitución», en las variables dureza, elasticidad y masticabilidad no se observaron diferencias significativas. La cohesividad y el volumen del pan resultaron significativamente menores en el pan con $36 \%$ de sustitución para cualquier proceso de elaboración.

En cuanto al color, se observó efecto significativo de la interacción en las variables: luminosidad de los panes (L), (a) e índice de blancura (WI). En L y WI todos los tratamientos resultaron estadísticamente significativos y con el mismo orden de medias, de mayor a menor: P1-36, P2-36, P1-20, P2-20. El orden de las medias responde al nivel de sustitución, siendo $36 \%$ el de menor luminosidad y WI, y los procesos siguen el orden P1 con menor luminosidad que P2. Respecto al 20\% de sustitución de harina compuesta, la variable a presentó diferencias significativas en los panes con $36 \%$, con un matiz de color más cercano al rojo. En el porcentaje de sustitución del $20 \%$ se observó un efecto del proceso, por lo que el proceso $\mathrm{P} 1$ resultó con matices más rojos que P2 (Tabla 3).

En el seguimiento de la textura del pan durante el almacenamiento los Gráficos 1, 2, 3 y 4 expresan las tendencias de los resultados obtenidos. En el Gráfico 1 se observa que el pan elaborado según el proceso P1 presentó un aumento más marcado de la dureza durante los cuatro días de seguimiento en relación al P2. En cuanto a los parámetros de elasticidad y cohesividad, se observó para ambos tipos de pan una disminución de los valores con el tiempo. Ocurrió una leve disminución de la masticabilidad en todos los tratamientos, con diferencias significativas entre el proceso y porcentaje de sustitución.

Si bien en los gráficos se observan tendencias de respuesta de los tratamientos en el tiempo, los datos no se ajustaron a un modelo de regresión lineal y se investigó otra forma de análisis de datos. Para ello se midió la posible autocorrelación de los datos experimentales a través del tiempo de estudio, ajustándose un modelo de medidas repetidas en el tiempo bajo tres condiciones de autocorrelación: autorregresiva de primer orden (AR1), Simétrica Compuesta (CS) y de Independencia. Estas tres condiciones fueron comparadas mediante los índices de Akaike Corregido (AICC) y el Índice Bayesiano corregido (BIC). Los resultados indicaron un mejor comportamiento del modelo de independencia entre observaciones, siendo los valores de AICC y BIC menores (160,4 y 160,5, respectivamente) frente a las condiciones de AR1 y CS (163,4 y 163,6, respectivamente, e iguales para los dos modelos).

Considerando los días de medida como repeticiones independientes, el pan elaborado según el procedimiento $\mathrm{P} 1$ fue significativamente más duro y de mayor masticabilidad. En las medidas de elasticidad y cohesividad no se observaron diferencias significativas según el proceso. De acuerdo al porcentaje de sustitución, se observaron diferencias significativas entre los niveles en masticabilidad y en el resto de las variables no se observaron diferencias significativas (Tabla 5).

En la Tabla 5 se presenta el estudio de la interacción entre los factores. Los valores de dureza de la miga de pan cuando se aplica el proceso P1 no se diferenciaron entre sí según el nivel de sustitución y fueron significativamente mayores a los panes elaborados según el proceso P2. Sin embargo, la dureza del pan elaborado según el proceso $\mathrm{P} 2$ se diferenció según el nivel de sustitución, aumentando con este.

\section{Conclusiones}

El proceso recomendado (P2) para la elaboración de pan con harinas compuestas mejora significativamente la calidad del pan y estas mejoras se mantienen en el tiempo. El proceso genera panes significativamente menos duros, más elásticos, más cohesivos, de menor masticabilidad y mayor volumen. El proceso P2 también afecta el índice de blancura del pan, siendo los panes elaborados según el proceso habitual (P1) más oscuros que los elaborados según el proceso P2.

El nivel de sustitución de harina precocida por extrusión afecta significativamente el volumen y el color del pan. Se observó una notoria disminución del volumen de pan con el $36 \%$ de sustitución, resultados concordantes con la bibliografía de referencia (Indrani, et al., 2010 y 2011; Gómez, et al., 2011). El pan con mayor nivel de sustitución (36\%) es más oscuro y menos luminoso.

\section{Reconocimientos}

A la Agencia Nacional de Investigación e Innovación (ANII) por apoyar la investigación con un $60 \%$ de subsidio. 


\section{Referencias}

AACC International, 1999. Approved methods of analysis. 10a ed. St. Paul: AACC Internacional. Method 44-40.

AACC International, 1999. Approved methods of analysis. 10a ed. St. Paul: AACC Internacional. Method 46-30.01.

American Oil Chemist's Society, 2009. Official method and recommended practices of the AOCS. 6a. ed. Urbana: AOCS. Official Method Bc 2-49.

American Oil Chemist's Society, 2009. Official method and recommended practices of the AOCS. 6a. Ed. Urbana: AOCS. Official Method Bc 3-49.

Anderson, R. A., Conway, H. F, Pfeifer, V. F. y Griffin E. L., Jr., 1969. Roll and extrusion cooking of grain sorghum grits. En: Cereal Science Today, 14, pp.372-375.

AOAC International, 2010. Official methods of analysis of AOAC International. 18a. ed. Gaithersburg: AOAC. Official Method 985.29.

Athar, N., Hardacre, A., Taylor, G., Clark, S., Harding, R. y McLaughlin, J., 2006. Vitamin retention in extruded food products. En: Journal of Food Composition and Analysis, 19, pp.379-383.

Brennan, Ch., Brennan, M., Derbyshire, E., Tiwari, B.K., 2011. Effects of extrusion on the polyphenols, vitamins and antioxidant activity of foods. En: Trends in Food Science Technology, 22(10), pp.1-6.

Burgess- Champoux, T., Marquart, L., Vickers, Z. y Reicks, M., 2006. Perceptions of children, parents, and teachers regarding whole-grain foods, and implications for school-based intervention. En: J. Nutr. Educ. Behav, 38, pp.230-237.

Bourne, Malcolm C., 2002. Food texture and viscosity: concept and measurement. Melbourne: Academic Press. (Food Science and Technology. International Series).

Crosa, M. J., Curia, A., Curutchet, A., Cadenazzi, M., Dotta, G., Ferreyra, V., Maidana, F, Souto, L. y Escudero, J., 2013. Estudio comparativo de la estabilidad lipídica de harinas de soja, sorgo, avena, salvado y germen de trigo con y sin extrusión. En: INNOTEC, (8), pp.30-38.

Ellis, J., Johnson, M.A., Fischer, J.G. y Hargrove, J. L., 2005. Nutrition and health education intervention for whole grain foods in the Georgia older Americans nutrition programs. En: J. Nutr. Elder, 24, pp.67-83.

Feillet, P., Guinet, R., Morel, M. H., Rouau, X., 1996. La masa, formación y desarrollo. En: Godon, Bernard, Guinet, Roland. La panificación aspectos socioeconómicos, materias primas, agentes de fermentación, tecnología, calidad. Barcelona: Montagud. pp.245-295.

Gómez, M., González, J., Oliete, B., 2011. Effect of extruded wheat germ on dough rheology and bread quality. En: Food Science and Technology, 44, pp.2231-2237.

Hansen, L., y Rose, M. S., 1996. Sensory acceptability is inversely related to development of fat rancidity in bread made from stored flour. En: J. Am Diet. Assoc., 96, pp.792-793.

HSU, C. L., Chen, W., Weng, Y. M. y Tseng, C. Y., 2003. Chemical composition, physical properties, and antioxidant activities of yam flours as affected by different drying methods. En: Food Chemistry, 83, pp.85-92.

Indrani, D., Soumya, C., Rajiv, J. y Venkateswara Rao, G., 2010. Multigrain Bread- Its dough rheology, microestructure, quality and nutritional characteristics. En: Journal of texture studies, 41, pp.302-319.
Indrani, D., Swetha, P., Soumya, C., Rajiv, J. Venkateswara Rao, G., 2011. Effect of multigrains on rheological, microestructural and quality characteristics of north Indian parotta-An indian flat bread. LWT. En: Food Science and Technology, 44, pp.719-724.

Instituto Nacional de Estadística, 2005. Los alimentos y las bebidas en los hogares. Encuesta Nacional de Gastos e Ingresos de los Hogares 2005-2006. Montevideo: INE.

International Organization For Standardization (Suiza), 2010. ISO 2171: Cereales, legumbres y subproductos. Determinación del rendimiento de cenizas por incineración. Ginebra: ISO.

Killeit, U., 1994. Vitamin retention in extrusion cooking. En: Food Chemistry, 49, pp.149-155.

Kuznesof, S., Brownlee, L.A., Moore, C., et al., 2012. Whole heart study participant acceptance of whole grain foods. En: Appetite, 59, pp.187-193.

Kyro, C., Skeie, G., Dragted, L.O., et al., 2012. Intake of wholw grain in Scandinavia: intake, sources and compliance with new national recommendations. En: Scand. J. Public Health, 40, pp.76-84.

Lafferty, A., Marquart, L. y Reicks, M., 2006. Hunting for whole grains: a supermarket tour. En: J. Nutr. Educ. Behav, 38, pp.197-198.

Marquart, L., Jonnalagadda, S.S., Van Klinken, B. J-W., Clemens, R., Jensen, G., Arndt, E. y Webb, D., 2013. En: Food Technology, 07(13), pp.52-62.

Matos-Chamorro, A. y Chambilla-Mamani, E., 2010. Importancia de la fibra dietética, sus propiedades funcionales en la alimentación humana y en la industria alimentaria. En: Revista de Investigación en Ciencia y Tecnología de Alimentos, 1(1), pp.4-17.

OIM (Institute of Medicine), 2011. Leveraging food technology for obesity prevention and reduction efforts workshop summary. Washington: National Academies Press.

Pérez, A., Drago, S. R., Carrara, C.R., De Greef, D., Torres, R. y González, R. J., 2008. Extrusion cooking of a maize/ soybean mixture: factors affecting expanded product characteristics and flour dispersion viscosity. En: Journal of Food Engineering, 87, pp.333-340.

Pomeranz, Y., 1992. Biochemical, functional, and nutritive changes during storage. En: Sauer, D. B., ed. Storage of cereal grains and their products. $4 \mathrm{a}$ ed. Florida: AACC International. pp.55-141.

Robin, F., Schuchmann, H. P., Palzer S. y Stefan, 2012. Dietary fiber in extruded cereals: limitations and opportunities. En: Trends in Food Science \& Technology, 28, pp.23-32.

Rose, D. J., Ogden, L., Dunn, M.L. y Pike, O.A., 2008. Enhanced lipid stability in whole wheat flour by lipase inactivation and antioxidant retention. En: Cereal Chemistry, 2, pp.218-223.

Sing, S., Gamlath, S. y Wakeling, L., 2007. Nutritional aspects of food extrusion: a review. En: International Journal of Food Science and Technology, 42, pp.916-929.

Sosa Moguel, O., Ruiz-Ruiz, J., Martinez-Ayala, A., Gonzalez, R., Drago, S., Betancur-Ancona, D. y Chel-Guerrero, L. 2009. Effect of extrusion conditions and lipxygenase inactivation treatment on physical and nutritional properties of corn/cowpea (Vigna unguiculata) blends. En: International Journal of Food Sciences and Nutrition, 60(S7), pp.341-354. 
Tait, S.P. y Galliard, T., 1988. Effect of baking quality of changes in lipid composition during wholemeal storage. En: Journal of Cereal Science, 8, pp.125-137.

Thane, C.W., Jones, A.R., Stephen, A.M., Seal, C. J. y Jebb, S.A., 2007. Comparative whole -grain intake of British adults in 1986-7 and 2000 - 1. En: Br. J. Nutr., 97, pp.987-992.

Turgeon, S., Rioux, L., 2011. Food matrix impact on macronutrients nutritional properties. En: Food Hydrocolloids, 25, pp.1915-1924.
U.S. Department of Agriculture y U.S. Department of Health and Human Services. Dietary guidelines for americans, 2010. 7a ed. Washington: U.S. Government Printing Office. pp. 29-30.

Vetrimani, R., Haridas, R., 1990. Studies on stabilization of wheat bran. En: Journal of Food Science and Technology, 27, pp.332-335. 